\title{
Luminosity Loss due to Beam Distortion and the Beam-Beam Instability*
}

\author{
Juhao $\mathrm{Wu}^{\dagger}$, T.O. Raubenheimer, A.W. Chao, A. Seryi, SLAC, Menlo Park, CA 94025, USA \\ C.K. Sramek, Rice University, Houston, TX 77251, USA
}

\begin{abstract}
In a linear collider, sources of emittance dilution such as transverse wakefields or dispersive errors will couple the vertical phase space to the longitudinal position within the beam (the so-called 'banana effect'). When the Intersection Point (IP) disruption parameter is large, these beam distortions will be amplified by a single bunch kink instability which will lead to luminosity loss. We study this phenomena both analytically using linear theory and via numerical simulation. In particular, we examine the dependence of the luminosity loss on the wavelength of the beam distortions and the disruption parameter. This analysis may prove useful when optimizing the vertical disruption parameter for luminosity operation with given beam distortions.
\end{abstract}

\section{Introduction}

To achieve the desired luminosity in a future linear collider, the beams are focused to small spot sizes and the resulting beam-beam forces can be very large. With oppositely charged beams, the beam-beam forces will lead to a mutual focusing or pinch which further increases the beam densities and the luminosity and is referred to as the luminosity enhancement. In addition, if the beams are offset from each other, the attractive beam-beam force can bring the beams closer together possibly recovering some of the lost luminosity. Unfortunately, if the beam-beam force is too large, this attraction can lead to an instability much like a plasma two-stream instability which is referred to as a single bunch kink instability [1].

The beam-beam force and resulting kink instability can be parameterized with the disruption parameter: $D_{x(, y)} \equiv$ $\sigma_{z} /\left[f_{x(, y)}\right]=2 N_{b} r_{e} \sigma_{z} /\left[\gamma \sigma_{x(, y)}\left(\sigma_{x}+\sigma_{y}\right)\right]$, where $f_{x(, y)}$ is the focal length due to the beam-beam force; $\sigma_{x(, y, z)}$ is the rms beam size, $N_{b}$ the number of particle per beam, $r_{e}$ the electron classical radius, and $\gamma$ the Lorentz factor. Normally, for a flat beam, $D_{x} \ll 1$, and hence the beam acts as a thin lens in the horizontal plane. In contrast, $D_{y} \gg 1$ and the two beams begin to oscillate in the vertical plane during the collision with the number of oscillations given by $n \approx 0.15 \sqrt{D_{y}}$. In simulations with $D_{y} \leq 10$, the beam-beam force is observed to reduce the luminosity loss due to centroid offsets, however for larger $D_{y}$ the luminosity becomes increasingly sensitive to small offsets [2]. In this paper, we investigate the impact of internal distortions in the beam. The many sources of emittance dilution lead to vertical position and angle offsets $\left\{y_{w}(z), y_{w}^{\prime}(z)\right\}$ of the slice centers as a function of their longitudinal position $z$

\footnotetext{
* Work supported by the U.S. Department of Energy under Contract No. DE-AC02-76SF00515.

$\dagger$ jhwu@SLAC.Stanford.EDU
}

in the beam. It can be expected that the two-stream instability will resonantly amplify distortions with wavelengths that are close to resonance.

\section{Equations of Motion}

Suppose that two beams move towards each other. For simplicity, their longitudinal distribution is assumed to be uniform, and their transverse distribution to be Gaussian. Assume that the beams collide with a relative vertical displacement and each beam is assumed to have the same number of particles per unit length $\lambda$ but with opposite charge. Assuming no redistribution of charges occurs during the collision, the Lorentz force near the axis is [4]

$$
F_{y, i n c} \approx \frac{4 \lambda e^{2}}{4 \pi \epsilon_{0}} \frac{Y_{d}}{\sigma_{y}\left(\sigma_{x}+\sigma_{y}\right)},
$$

where $e$ is the elementary charge, $\epsilon_{0}$ the vacuum permittivity, $Y_{d}$ the particle vertical displacement relative to the centroid of the other beam. We are interested in the luminosity loss due to the variation of the beam centroid along the bunch; hence, we need to integrate over the transverse distribution. Assuming small offsets of the two beams, this reduces the effective incoherent force in Eq. (1) by a factor of 2 to get the so-called coherent force of $F_{y, \text { coh }} \approx F_{y, \text { inc }} / 2$. We study cold beams, and the equations of motion for the two beams, moving with velocity $v$, read [1]

$$
\left(\frac{\partial}{\partial t} \pm v \frac{\partial}{\partial s}\right)^{2} y_{l(, r)}=-\frac{2 \lambda r_{e} c^{2}\left[y_{l(, r)}-y_{r(, l)}\right]}{\sigma_{y}\left(\sigma_{x}+\sigma_{y}\right) \gamma}
$$

where, $y_{l(, r)}$ is the centroid displacements of the electron (positron) beam from the reference axis. In the above model, the evolution of the beam envelope due to the pinch effect is not included. However, since the beam-beam instability dominates, this simplification is quite good, as evidenced by the comparison with simulation in the following.

\section{Initial Value Problem}

The internal coordinate $z$ is introduced to label the slice at a distance $z$ from the head of the beam, and $0<z<l$, where $l$ is the beam length. We define $t=0$ when the heads of the two beams collide. We also define $s=0$ as the IP where the two beams first collide. The positive $s$ direction is to the right. Hence in the left-coming beam, the slice $z$ will be located at $s=v t-z$ at time $t$ when the beam head is at the location $s=v t$. In the right-coming beam, we also introduce $z$ to describe the distance between a certain slice and the head of the beam, and again $0<z<l$. Hence, when the head of the right-coming beam is at location $s=$ $-v t$ at time $t$, the slice $z$ is at location $s=-v t+z$. We now 
use $(s, z)$ as the independent variable pair, and we define $k_{0}^{2} \equiv 2 \lambda r_{e} /\left[\sigma_{y}\left(\sigma_{x}+\sigma_{y}\right) \gamma\right]$. Please refer to Fig. 1 of Ref.

[5] for definition of the coordinate system.

Now let us study the case where the right-coming beam has an initial sinusoidal modulation, and the left-coming beam is undistorted and on-axis. The initial conditions are then

$$
y_{l}(0, z)=0 \text { and }\left.\frac{\partial y_{l}(s, z)}{\partial s}\right|_{s=0}=0,
$$

for the left-coming electron beam. Similarly,

$$
y_{r}(0, z)=y_{r 0} \sin (k z) \text { and }\left.\frac{\partial y_{r}(s, z)}{\partial s}\right|_{s=0}=0,
$$

for the right-coming positron beam. The equations of motion together with the initial conditions yield the following integral representation of the solution

$$
\begin{aligned}
y_{l}(s, z) & =y_{l}(z / 2, z) \cos \left[k_{0}(s-z / 2)\right] \\
& +\left.\frac{\partial y_{l}(s, z)}{\partial s}\right|_{s=z / 2} \frac{\sin \left[k_{0}(s-z / 2)\right]}{k_{0}} \\
& +k_{0} \int_{z / 2}^{s} d s^{\prime} y_{r}\left(-s^{\prime}, 2 s^{\prime}-z\right) \sin \left[k_{0}\left(s-s^{\prime}\right)\right]
\end{aligned}
$$

Similarly, for the right-coming beam, we have

$$
\begin{aligned}
y_{r}(s, z) & =y_{r}(-z / 2, z) \cos \left[k_{0}(s+z / 2)\right] \\
& +\left.\frac{\partial y_{r}(s, z)}{\partial s}\right|_{s=-z / 2} \frac{\sin \left[k_{0}(s+z / 2)\right]}{k_{0}} \\
& +k_{0} \int_{-z / 2}^{s} d s^{\prime} y_{l}\left(-s^{\prime},-2 s^{\prime}-z\right) \sin \left[k_{0}\left(s-s^{\prime}\right)\right] .
\end{aligned}
$$

\section{Series Solution}

Let us solve the above set of Eqs. (5) and (6) via a series solution approach $[6,7]$. We expand $y_{r(, l)}(s, z)$ in a series of powers in $k_{0}$

$$
y_{r(, l)}(s, z)=\sum_{n=0}^{\infty} y_{r(, l)}^{(n)}(s, z)
$$

and obtain the $n$ th-order term from the $(n-1)$ th-order term. According to Eq. (5), for $n=1,2,3, \cdots, y_{l}(s, z)$ would be

$$
y_{l}^{(n)}(s, z)=k_{0} \int_{z / 2}^{s} d s^{\prime} y_{r}^{(n-1)}\left(-s^{\prime}, 2 s^{\prime}-z\right) \sin \left[k_{0}\left(s-s^{\prime}\right)\right]
$$

and similarly, according to Eq. (6), for $y_{r}(s, z)$, we have

$$
y_{r}^{(n)}(s, z)=k_{0} \int_{-z / 2}^{s} d s^{\prime} y_{l}^{(n-1)}\left(-s^{\prime},-2 s^{\prime}-z\right) \sin \left[k_{0}\left(s-s^{\prime}\right)\right] .
$$

General Solution According to Eqs. (5) and (6) with the initial conditions in Eqs. (3) and (4), we get, $y_{r}^{(0)}(s, z)=y_{r 0} \sin (k z) \cos \left[k_{0}(s+z / 2)\right]$, and $y_{l}^{(0)}(s, z)=0$. Noticing that, in the limit of $k_{0} \rightarrow 0$, we have $y_{r}^{(0)}(s, z)=y_{r 0} \sin (k z)$. This is just the initial modulation profile.

For a large $D_{y}$, which leads to many oscillations during the collision, we are eligible to consider the limit of $k_{0} s \gg$ 1 and also $k s \gg 1$. Therefore, we keep only the largest terms in $k_{0} s$ and $k s$. The asymptotic solutions are:

$$
\begin{aligned}
y_{r}(s, z) & \approx i y_{r 0} \frac{k k_{0}}{k_{0}^{2}-4 k^{2}} \sqrt{\frac{|2 s+z|}{z}} \\
& \times J_{1}\left(i k_{0} \sqrt{z|2 s+z|} / 2\right) \cos \left[k_{0}(s+z)\right]
\end{aligned}
$$

for $-z / 2>s>-(l+z) / 2$; and

$$
\begin{aligned}
y_{l}(s, z) & \approx-y_{r 0} \frac{k k_{0}}{k_{0}^{2}-4 k^{2}} \\
& \times \quad J_{0}\left(i k_{0} \sqrt{z(2 s-z)} / 2\right) \sin \left[k_{0}(s-z)\right]
\end{aligned}
$$

for $(l+z) / 2>s>z / 2$. Notice that, the solutions given in Eqs. (10) and (11) have a singular point at $k_{0}=2 k$, which indicates a resonance behavior studied in the following.

Resonance Case At resonance, $k_{0}=2 k$, the recursion relation of Eq. (8) and (9) should be revised by replacing $k_{0}$ with $2 k$. The asymptotic solutions are:

$$
y_{r}(s, z) \approx \frac{y_{r 0}}{2} J_{0}(i k \sqrt{z|2 s+z|}) \sin [2 k(s+z)],
$$

for $-z / 2>s>-(l+z) / 2$; and

$$
y_{l}(s, z) \approx \frac{i y_{r 0}}{2} \sqrt{\frac{2 s-z}{z}} J_{1}(i k \sqrt{z(2 s-z)}) \cos [2 k(s-z)]
$$

for $(l+z) / 2>s>z / 2$.

\section{Luminosity}

The luminosity is defined as [8]

$$
\mathcal{L}=2 N_{b}^{2} v \int d x d y d s d t n_{l}\left(x, y, z_{l}, t\right) n_{r}\left(x, y, z_{r}, t\right),
$$

where $z_{l}=v t-s$ and $z_{r}=v t+s$ and we have assumed the same number population $N_{b}$ in each beam and head-on collisions. The distribution function is normalized to unit, i.e., $\int d x d y d s n_{l(, r)}\left(x, y, z_{l(, r)}, t\right)=1$. Assuming Gaussian transverse distributions and a uniform longitudinal distribution, and ignoring the luminosity enhancement due to beam-beam pinch, the 'geometric' luminosity is $\mathcal{L}_{00}=N_{b}^{2} /\left[4 \pi \sigma_{x} \sigma_{y}\right]$. Finally, the nominal luminosity $\mathcal{L}_{0}$, including the effect of the luminosity enhancement, is found by multiplying by the enhancement factor $H_{D}$ which is typically between 1 and 2 for flat beam collisions, i.e., $\mathcal{L}_{0}=\mathcal{L}_{00} H_{D}$. Now, let us study the luminosity loss due to the beam-beam disruption. Given the analytical general solutions in Eqs. (10) - (13), we can numerically compute the luminosity. On the other hand, we also simulate the luminosity loss via GuineaPig [9] for longitudinal uniform distribution and Gaussian distribution as well. 
Table 1: Summary of the parameters for the US Cold [3].

\begin{tabular}{|c|c|c|}
\hline$E(\mathrm{GeV})$ & $N_{b}\left(10^{10}\right)$ & $\sigma_{x}(\mu \mathrm{m})$ \\
\hline 250 & 2.0 & 0.543 \\
\hline$\sigma_{x^{\prime}}(\mu \mathrm{rad})$ & $\sigma_{y}(\mathrm{~nm})$ & $\sigma_{y^{\prime}}(\mu \mathrm{rad})$ \\
\hline 36 & 5.7 & 14 \\
\hline$\sigma_{z}(\mathrm{~m} \mathrm{~m})$ & $\sigma_{\delta}(\%)$ & $D_{y}$ \\
\hline 0.3 & 0.1 & 22.0 \\
\hline
\end{tabular}

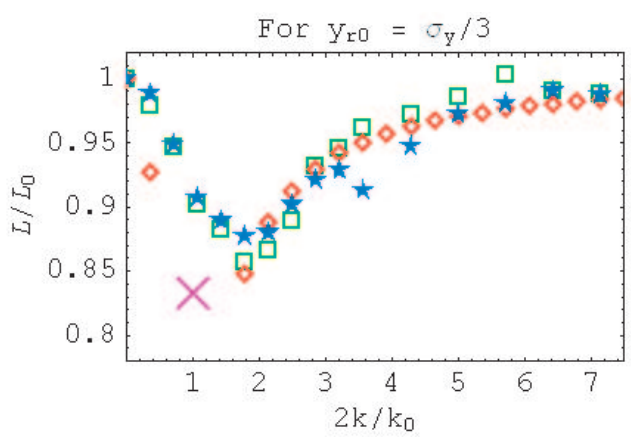

Figure 1: The $\mathcal{L} / \mathcal{L}_{0}$ as a function of $2 k / k_{0}$. The "square" is the simulation for truncated (at $\pm \sqrt{3} \sigma_{z}$ ) Gaussian distribution; the " $\star$ " is the simulation for a uniform distribution; the " $\diamond "$ is the result with solutions in Eqs. (10) and (11); the " $x$ " is the result with solutions in Eqs. (12) and (13).

\section{Results}

Now let us illustrate how the beam-beam effect leads to a large luminosity loss, even if the emittance is not degraded much. We study the ILC US Cold [3] with the parameters in Table 1. Shown in Fig. 1, we find that for $y_{r 0}=\sigma_{y} / 3$, the beam-beam interaction leads to a resonance where 14 $\%$ luminosity loss is observed. Now, if one does not notice the instability studied in this paper, then he will find 3 $\%$ emittance growth, which leads to only $1 \%$ "geometric" luminosity loss simply due to the initial distortion. Hence, the geometric emittance growth in this case is not a good measure for the luminosity loss [10]. Similar situation is found for different $y_{r 0}$. In Figs. 2, we show the luminosity

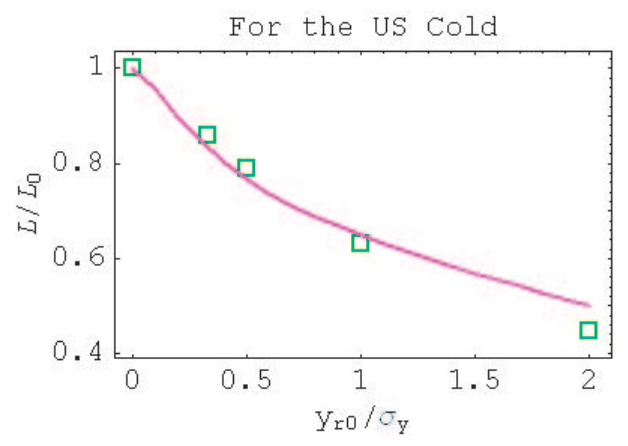

Figure 2: The $\mathcal{L} / \mathcal{L}_{0}$ as a function of $y_{r 0} / \sigma_{y}$ with "square" having same meaning as in Fig. 1 and the curve is the result with solutions in Eqs. (12) and (13).

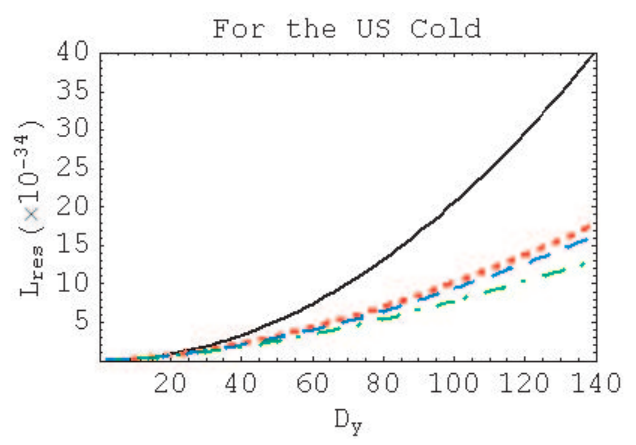

Figure 3: The resonance luminosity $\mathcal{L}_{\text {res }}$ as a function of $D_{y}$. The dotted, dashed, and dotted-dashed curves stand for the cases where $y_{r 0} / \sigma_{y}=1 / 3,1 / 2$, and 1 , respectively. As a comparison, the solid curve stands for the case with no modulation, i.e., $\mathcal{L}_{0}$, or $y_{r 0}=0$.

loss as a function of $y_{r 0}$. Once again, very good agreement between the analytical result and the simulation is found.

The study shows that the luminosity loss computed analytically agrees with the simulation results quite well. The results for a longitudinal uniform distribution is very close to those for a Gaussian distribution. The study also indicates that this luminosity loss due to the beam-beam interaction is very sensitive to the disruption parameter $D_{y}$. To illustrate this dependency, we plot in Fig. 3, the luminosity at resonance, i.e., with solution given in Eqs. (12) and (13), as a function of $D_{y}$ which relates to $k_{0}(=2 k)$. In our study, we vary $N_{b}$ alone to vary $D_{y}$. Without the beambeam instability considered in this paper, increasing the disruption parameter $D_{y}$ will increase the luminosity $\mathcal{L}_{0}$ quadratically. On the other hand, the beam-beam instability introduces a large luminosity loss when $D_{y}$ increases. This leads to the phenomena that beyond certain value of $D_{y, c}$ ( $D_{y, c} \sim 20$ in our paper), increasing $D_{y}$ will no longer efficiently increase the luminosity. To conclude, the luminosity loss due to beam-beam instability is substantial, and needs serious consideration in future ILC design.

\section{REFERENCES}

[1] Y.H. Chin, DESY 87-011, Jan. 1987.

[2] K. Yokoya and P. Chen, Lect. Notes in Phys., 400, 415 (1992).

[3] http://www.slac.stanford.edu/xorg/accelops/.

[4] I.M. Kapchinskij and V.V. Vladimirskij, Proc. Intern. Conf. High Energy Accel. and Instr., (CERN, 1959), p. 274.

[5] J. Wu and T.O. Raubenheimer, this proceedings.

[6] A.W. Chao et al., Nucl. Instrum. and Methods, 178, 1 (1980).

[7] T.O. Raubenheimer, F. Zimmermann, PRE 52, 5487 (1995).

[8] C. Møller, K. Danske Vidensk. Selsk. Mat.-Fys. Medd. 23, 1 (1945).

[9] D. Schulte, Ph.D. thesis, University of Hamburg 1996.

[10] R. Brinkmann et al., PAC 2001, p. 2020. 